PREPARED FOR THE U.S. DEPARTMENT OF ENERGY, UNDER CONTRACT DE-AC02-76CH03073

PPPL-3743

PPPL-3743

UC-70

Guiding Center Equations in Toroidal Equilibria

by

Roscoe White and Leonid Zakharov

September 2002

$N_{\substack{\text { PRInCETOn PLASMA } \\ \text { PHYSIES LABORATORY }}}^{D}$

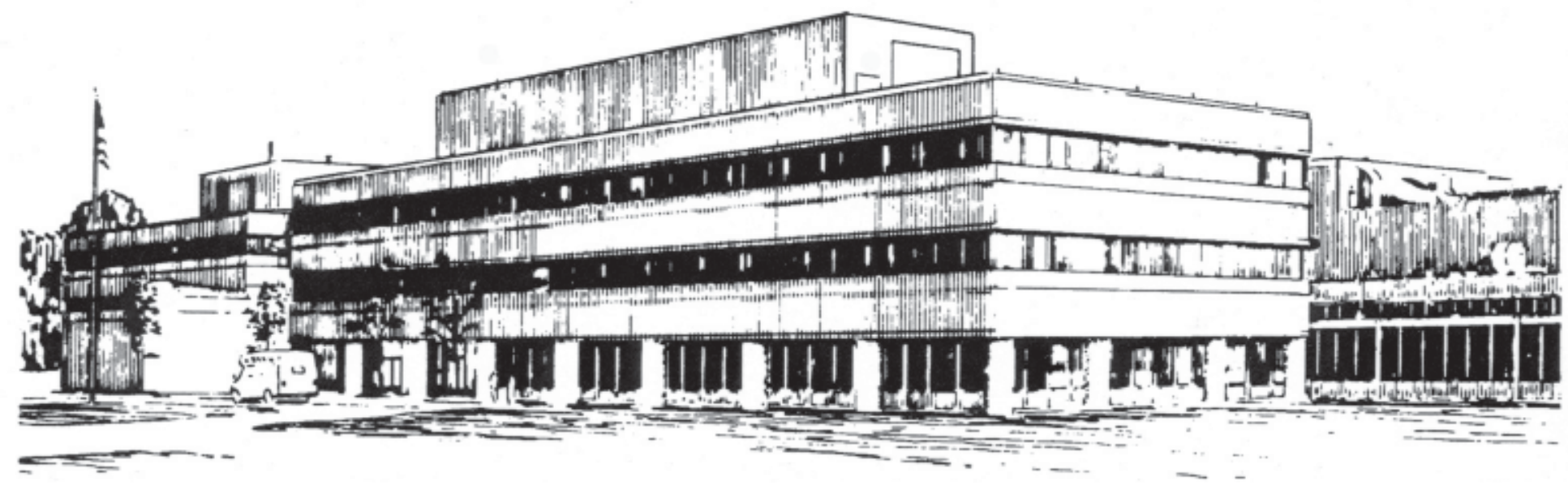

PRINCETON PLASMA PHYSICS LABORATORY PRINCETON UNIVERSITY, PRINCETON, NEW JERSEY 


\section{PPPL Reports Disclaimer}

This report was prepared as an account of work sponsored by an agency of the United States Government. Neither the United States Government nor any agency thereof, nor any of their employees, makes any warranty, express or implied, or assumes any legal liability or responsibility for the accuracy, completeness, or usefulness of any information, apparatus, product, or process disclosed, or represents that its use would not infringe privately owned rights. Reference herein to any specific commercial product, process, or service by trade name, trademark, manufacturer, or otherwise, does not necessarily constitute or imply its endorsement, recommendation, or favoring by the United States Government or any agency thereof. The views and opinions of authors expressed herein do not necessarily state or reflect those of the United States Government or any agency thereof.

\section{Availability}

This report is posted on the U.S. Department of Energy's Princeton Plasma Physics Laboratory Publications and Reports web site in Fiscal Year 2002. The home page for PPPL Reports and Publications is: http://www.pppl.gov/pub_report/

DOE and DOE Contractors can obtain copies of this report from:

U.S. Department of Energy

Office of Scientific and Technical Information

DOE Technical Information Services (DTIS)

P.O. Box 62

Oak Ridge, TN 37831

Telephone: (865) 576-8401

Fax: (865) 576-5728

Email: reports@adonis.osti.gov

This report is available to the general public from:

National Technical Information Service

U.S. Department of Commerce

5285 Port Royal Road

Springfield, VA 22161

Telephone: 1-800-553-6847 or

(703) 605-6000

Fax: (703) 321-8547

Internet: http://www.ntis.gov/ordering.htm 


\title{
Guiding center equations in toroidal equilibria
}

\author{
Roscoe White, Leonid Zakharov \\ Plasma Physics Laboratory, Princeton University, P.O.Box 451, \\ Princeton, New Jersey 08543
}

\begin{abstract}
Guiding center equations for particle motion in a general toroidal magnetic equilibrium configuration are derived using magnetic coordinates. Previous derivations made use of Boozer coordinates, in which the poloidal and toroidal angle variables are chosen so that the Jacobian is inversely proportional to the square of the magnetic field. It is shown that the equations for guiding center motion in any equilibrium possessing nested flux surfaces have exactly the same simple form as those derived in this special case. This allows the use of more spatially uniform coordinates instead of the Boozer coordinates, greatly increasing the accuracy of calculations in large beta and strongly shaped equilibria.
\end{abstract}

PACS numbers: 52.20.Dq 52.65.Cc 
High energy particles in toroidal thermonuclear fusion devices, such as fusion product alpha particles, or those due to neutral beam injection or ion cyclotron heating, are well known to behave as independent test particles, and their confinement can be analyzed to a high degree of accuracy using the equilibrium field and the guiding center approximation. Guiding center equations are important for simulations requiring the calculation of particle motion for long periods of time, such as the study of alpha particle confinement during the time it takes for the particles to slow to the energy of the background plasma. It is advantageous to use magnetic coordinates for such calculations, since the particle motion is to lowest order along the magnetic field, and only to higher order (the drift motion) across the field.

In general toroidal coordinates $\psi_{p}, \theta, \zeta^{\prime}$ consider a magnetic field which possesses nested toroidal flux surfaces labeled by $\psi_{p}$, with $\theta$ a poloidal angle, and $\zeta^{\prime}$ a toroidal angle. Since $\vec{B}$ is orthogonal to $\nabla \psi_{p}$ write

$$
\vec{B}=\nabla \psi_{p} \times \nabla V
$$

and choose $V=q\left(\psi_{p}\right) \theta-\zeta^{\prime}+\lambda\left(\psi_{p}, \theta, \zeta^{\prime}\right)$. Choose $2 \pi \psi_{p}$ to be the poloidal flux inside the magnetic surface. The element of surface area in a poloidal section is $d \vec{S}_{p}=\mathcal{J}_{p} \nabla \theta d \zeta^{\prime} d \psi_{p}$, giving

$$
2 \pi \psi_{p}=\int \vec{B} \cdot \nabla \theta \mathcal{J} d \psi_{p} d \zeta^{\prime}=\int d \psi_{p} d \zeta^{\prime}-\int_{\zeta_{0}^{\prime}}^{\zeta_{0}^{\prime}+2 \pi} \frac{\partial \lambda}{\partial \zeta} d \psi_{p} d \zeta^{\prime}
$$

for any $\zeta_{0}^{\prime}$, with the Jacobian given by $\mathcal{J}_{p}^{-1}=\nabla \psi_{p} \cdot \nabla \theta \times \nabla \zeta^{\prime}$, and thus $\lambda$ is periodic in $\zeta^{\prime}$. Replace $\zeta^{\prime}$ with $\zeta$ through $\zeta^{\prime}-\lambda=\zeta$ and note that adding $2 \pi$ to $\zeta$ is equivalent to adding $2 \pi$ to $\zeta^{\prime}$, and thus $\lambda$ is also periodic considered as a function of $\zeta$. In these "straight field line variables", $d \zeta / d \theta=\vec{B} \cdot \nabla \zeta / \vec{B} \cdot \nabla \theta=$ $q(\psi)$ along a field line, and we have the contravariant representation for $\vec{B}$

$$
\vec{B}=\nabla \psi \times \nabla \theta+\nabla \zeta \times \nabla \psi_{p}
$$

with $d \psi / d \psi_{p}=q(\psi)$. Now choose $2 \pi \psi$ to be the toroidal flux inside the surface. The element of surface area in a toroidal section is $d \vec{S}_{t}=\mathcal{J} \nabla \zeta d \theta d \psi$, giving

$$
2 \pi \psi=\int \vec{B} \cdot \nabla \zeta \mathcal{J} d \psi d \theta=\int d \psi d \theta+\int_{\theta_{0}}^{\theta_{0}+2 \pi} \frac{\partial \lambda}{\partial \theta} d \psi d \theta
$$


for any $\theta_{0}$, with the Jacobian given by $\mathcal{J}^{-1}=\nabla \psi \cdot \nabla \theta \times \nabla \zeta=q \mathcal{J}_{p}^{-1}$, and thus $\lambda$ is also periodic in $\theta$. The surface labels $\psi$ and $\psi_{p}$ can be used interchangeably, according to which is more convenient for the equilibrium under study. For example, in a tokamak the minor radius is a single valued function of both the toroidal and poloidal flux, but in a reversed field pinch it is a single valued function only of the poloidal flux.

From the equilibrium condition

$$
\vec{j} \times \vec{B}=\nabla p
$$

with $p=p(\psi)$ it follows that $\vec{j} \cdot \nabla \psi=0$. Write $\vec{j}=\nabla \psi \times \nabla W$, and choose $W=\bar{I}^{\prime}(\psi) \theta+\bar{g}^{\prime}(\psi) \zeta+\alpha(\psi, \theta, \zeta)$. Then

$$
\vec{j}=\left(\bar{I}^{\prime}(\psi)+\alpha_{\theta}^{\prime}\right) \nabla \psi \times \nabla \theta+\left(\bar{g}^{\prime}(\psi)+\alpha_{\zeta}^{\prime}\right) \nabla \psi \times \nabla \zeta
$$

with primes indicating derivatives with respect to the subscripted variable. Choose $2 \pi \bar{I}$ to be the toroidal current inside $\psi$, and $2 \pi \bar{g}$ to be the poloidal current outside $\psi$. Then as is the case with $\lambda, \alpha$ is periodic in $\theta, \zeta$. Then $\nabla \times \vec{B}=\vec{j}$ gives the covariant representation for $\vec{B}$

$$
\vec{B}=\delta \nabla \psi+I \nabla \theta+g \nabla \zeta
$$

with $I(\psi, \theta, \zeta)=\bar{I}(\psi)+\sigma_{\theta}^{\prime}$, and $g(\psi, \theta, \zeta)=\bar{g}(\psi)+\sigma_{\zeta}^{\prime}$, and $\delta(\psi, \theta, \zeta)=\sigma_{\psi}^{\prime}-\alpha$.

These three flux functions $q(\psi), \bar{g}(\psi), \bar{I}(\psi)$ and the two functions of three variables $\alpha(\psi, \theta, \zeta)$ and $\sigma(\psi, \theta, \zeta)$ are further related. Dot the covariant and contravariant expressions for $\vec{B}$, giving $B^{2} \mathcal{J} q=g q+I$. Averaging in $\zeta$ and $\theta$ we find $q\left(\mathcal{J} B^{2}\right)_{00}=\bar{g} q+\bar{I}$ with $f_{00}=\int_{0}^{2 \pi} \int_{0}^{2 \pi} f d \zeta d \theta /\left(4 \pi^{2}\right)$. We also obtain the magnetic differential equation for the 3-D function $\sigma, q \mathcal{J} B^{2}-$ $q\left(\mathcal{J} B^{2}\right)_{00}=q \sigma_{\zeta}^{\prime}+\sigma_{\theta}^{\prime}$. Now use the equilibrium condition, Eq. 5. Substituting the expression for $\vec{j}$ we find $\left(\bar{I}^{\prime}+\alpha_{\theta}^{\prime}\right)+\left(\bar{g}^{\prime}+\alpha_{\zeta}^{\prime}\right) q=\mathcal{J} p^{\prime} q$. Averaging in $\zeta$ and $\theta$ we find that $\bar{I}, \bar{g}$, and $p^{\prime}$ are related through $\bar{I}^{\prime}+\bar{g}^{\prime} q=\mathcal{J}_{00} p^{\prime} q$. Thus only two of the three functions $\bar{g}, q, \bar{I}$ can be independently chosen. Further we find the magnetic differential equation for the 3-D function $\alpha, \alpha_{\theta}^{\prime}+q \alpha_{\zeta}^{\prime}=\tilde{\mathcal{J}} p^{\prime} q$ with $\tilde{\mathcal{J}}=\mathcal{J}-\mathcal{J}_{00}$. Primes here indicate derivatives with respect to $\psi$. An equilibrium is thus deternined by two of the three flux functions $\bar{g}, \bar{I}, q$, and the two functions of three variables $\sigma$ and $\alpha$. Changing the flux variable to $\psi_{p}$ introduces factors of $q$ in the derivative terms and in the change from $\mathcal{J}$ to $\mathcal{J}_{p}$. 
The guiding center Hamiltonian is

$$
H\left(\rho_{\|}, \psi, \zeta, \theta\right)=\frac{\rho_{\|}^{2} B^{2}}{2}+\mu B+\Phi
$$

with $\mu$ the magnetic moment, $\rho_{\|}=v_{\|} / B$ the normalized "parallel gyro radius", and $\Phi$ the electric potential. The field magnitude $B$ and the potential may be functions of $\psi,\left(\psi_{p}\right), \theta$ and $\zeta$. The units are defined by the on-axis gyro frequency (time) and the major radius (distance).

The Lagrangian can be put in the form $[1,2,3]$

$$
L=\left[\psi+\rho_{\|} I\right] \dot{\theta}+\left[\rho_{\|} g-\psi_{p}\right] \dot{\zeta}+\mu \dot{\xi}-H,
$$

with $\xi$ the gyrophase, and the canonical coordinates are $\zeta$ and $\theta$ with the canonical momenta $P_{\zeta}=\rho_{\|} g-\psi_{p}$, and $P_{\theta}=\psi+\rho_{\|} I$.

Note that to achieve this form the guiding center velocity along a field line has been modified by a correction of the order of the gyroradius[3]. This change in the definition of the guiding center, essential to obtain the Hamiltonian form given, does not modify the particle orbit, but only the time it takes to complete the orbit. Without this modification the motion does not satisfy the Liouville equation, and for some configurations can even violate energy conservation.

The equations of motion are

$$
\begin{array}{ll}
\dot{\zeta}=\frac{\partial H}{\partial P_{\zeta}} & \dot{P}_{\zeta}=-\frac{\partial H}{\partial \zeta} \\
\dot{\theta}=\frac{\partial H}{\partial P_{\theta}} & \dot{P}_{\theta}=-\frac{\partial H}{\partial \theta}
\end{array}
$$

Previous derivations of equations for advancing the variables $\rho_{\|}, \psi, \zeta, \theta$, made use of Boozer coordinates[4]. In this work we show that this restriction of the coordinate system is not necessary.

The Hamiltonian is expressed in the variables $y_{k}=\rho_{\|}, \psi, \zeta, \theta$, but Hamilton's equations are given in $x_{k}=P_{\zeta}, P_{\theta}, \zeta, \theta$. Partial derivatives for this change of variables are

$$
\left|\frac{\partial x_{k}}{\partial y_{j}}\right|=\left|\begin{array}{cccc}
\partial_{\rho_{\|}} P_{\zeta} & \partial_{\psi} P_{\zeta} & \partial_{\zeta} P_{\zeta} & \partial_{\theta} P_{\zeta} \\
\partial_{\rho_{\|}} P_{\theta} & \partial_{\psi} P_{\theta} & \partial_{\zeta} P_{\theta} & \partial_{\theta} P_{\theta} \\
0 & 0 & 1 & 0 \\
0 & 0 & 0 & 1
\end{array}\right|
$$


The inverse is

$$
M \equiv\left|\frac{\partial y_{k}}{\partial x_{j}}\right|=\frac{1}{D}\left|\begin{array}{cccc}
\partial_{\psi} P_{\theta} & -\partial_{\psi} P_{\zeta} & T_{\psi \zeta} & T_{\psi \theta} \\
-\partial_{\rho_{\|}} P_{\theta} & \partial_{\rho_{\|}} P_{\zeta} & T_{\zeta \rho_{\|}} & T_{\theta \rho_{\|}} \\
0 & 0 & D & 0 \\
0 & 0 & 0 & D
\end{array}\right|
$$

where $T_{\gamma \beta}=\partial_{\gamma} P_{\zeta} \partial_{\beta} P_{\theta}-\partial_{\beta} P_{\zeta} \partial_{\gamma} P_{\theta}$. Note that the determinant $D=g q+I+$ $\rho_{\|}\left(g I_{\psi}^{\prime}-I g_{\psi}^{\prime}\right)$ involves derivatives of $I$ and $g$ with respect to the variable $\psi$, and none with respect to $\zeta$ or $\theta$. In a general equilibrium of course both $I$ and $g$ are functions of all three variables.

The equations of motion can be written

$$
\frac{d x_{k}}{d t}=S_{k j} \frac{\partial H}{\partial x_{j}}
$$

with

$$
S=\left|\begin{array}{cccc}
0 & 0 & -1 & 0 \\
0 & 0 & 0 & -1 \\
1 & 0 & 0 & 0 \\
0 & 1 & 0 & 0
\end{array}\right|
$$

which becomes in the variables $y_{k}$

$$
\frac{d y_{k}}{d t}=\left(M S M^{T}\right)_{k j} \frac{\partial H}{\partial y_{j}}
$$

or

$$
\left|\begin{array}{c}
\dot{\rho}_{\|} \\
\dot{\psi} \\
\dot{\zeta} \\
\dot{\theta}
\end{array}\right|=\frac{1}{D}\left|\begin{array}{cccc}
0 & 0 & -\partial_{\psi} P_{\theta} & \partial_{\psi} P_{\zeta} \\
0 & 0 & \partial_{\rho_{\|}} P_{\theta} & -\partial_{\rho_{\|}} P_{\zeta} \\
\partial_{\psi} P_{\theta} & -\partial_{\rho_{\|}} P_{\zeta} & 0 & 0 \\
\partial_{\psi} P_{\zeta} & \partial_{\rho_{\|}} P_{\zeta} & 0 & 0
\end{array}\right|\left|\begin{array}{c}
\partial_{\rho_{\|}} H \\
\partial_{\psi} H \\
\partial_{\zeta} H \\
\partial_{\theta} H
\end{array}\right|
$$

where we have used $\partial_{\zeta} P_{\theta}=\partial_{\theta} P_{\zeta}$. In this matrix there are no derivatives of the canonical momenta with respect to $\zeta$ or $\theta$, and thus no derivatives of $I$ or $g$ with respect to these variables appear in the equations of motion. The equations of motion then have exactly the same form as they have using 
Boozer coordinates[3], where $\sigma=0$ and $g=g(\psi), I=I(\psi)$. However this choice fixes both $\zeta$ and $\theta$ in terms of the equilibrium parameters, and can lead to numerically inconvenient variables. The complication of general coordinates consists in the fact that both $g$ and $I$ must be evaluated as functions of all three variables in a fully $3 \mathrm{D}$ equilibrium, or in axisymmetric equilibria, that $I=I(\psi, \theta)$.

We note in passing that these equations are easily generalized to include time dependent perturbations which destroy the magnetic flux surfaces, so the initial restriction of the existence of such surfaces is not a severe one.

We conclude that in a general equilibrium configuration with nested toroidal flux surfaces expressed in any straight field line coordinates the form of the equations of motion are exactly the same as those given using Boozer coordinates. This fact is very useful for practical application. In Figs. 1, 2 are shown the coordinate systems for an axisymmetric National Spherical Torus Experiment (NSTX) equilibrium[6] of $12 \%$ beta (the ratio of plasma pressure to magnetic pressure) using Boozer coordinates and using the more regular coordinates $r(\psi, \theta)=\sum r_{m}(\psi) e^{i m \theta}, z(\psi, \theta)=z_{0}(\psi)+z_{1}(\psi) \sin \theta$ used by the Equilibrium and Stability Code (ESC)[7]. These coordinates coincide with the Boozer coordinates near the magnetic axis but are much more regular near the plasma edge. Clearly in using Boozer coordinates it is difficult to obtain a good representation of the field quantities near the outboard midplane. Using the more uniform coordinates involves the numerical representation of two functions of two variables, $B(\psi, \theta)$ and $I(\psi, \theta)$ instead of only $B$, but this inconvenience is more than compensated by the increased accuracy of the representation. In Fig. 3 is shown the dependence of the magnetic field on $\theta$ for these two choices of coordinates, as well as the dependence of the function $I(\psi, \theta)$ on $\theta$, for five surfaces ranging from $\psi=0.55 \psi_{w}$ to $\psi_{w}$ with $\psi_{w}$ the outside bounding surface. The difficulty encountered numerically in using the Boozer representation is due to the sharp behaviour of $B$ at the outer midplane. Note that on the other hand $I(\psi, \theta)$ in the ESC coordinates is a very smooth function.

This work was supported by the U.S. Department of Energy Grant DE-FG03-94ER54271. 


\section{References}

[1] R.B. White, "The theory of toroidally confined plasmas", Imperial College Press, 2001.

[2] R.B. White and M. S. Chance, Phys. Fluids 272455 (1984).

[3] R.B. White, Phys. Fluids B 2(4), 845 (1990).

[4] A. H. Boozer, Phys. Fluids 241999 (1981).

[5] D. A. Garren and A. H. Boozer, Phys. Fluids B 3(10), 845 (1991).

[6] M. Ono et al., Nucl. Fusion 40, 557 (2000)

[7] L. Zakharov and A. Pletzer, Physics of Plasmas 6, 4693 (1999).

Figure Captions

Fig. 1 NSTX equilibrium with $\beta=12 \%$ using Boozer coordinates.

Fig. 2 NSTX equilibrium with $\beta=12 \%$, using ESC coordinates.

Fig. 3 Variation of B in Boozer and ESC coordinates, and $I(\psi, \theta)$ in ESC coordinates, on five flux surfaces ranging from $\psi=0.55 \psi_{w}$ to $\psi_{w}$. 


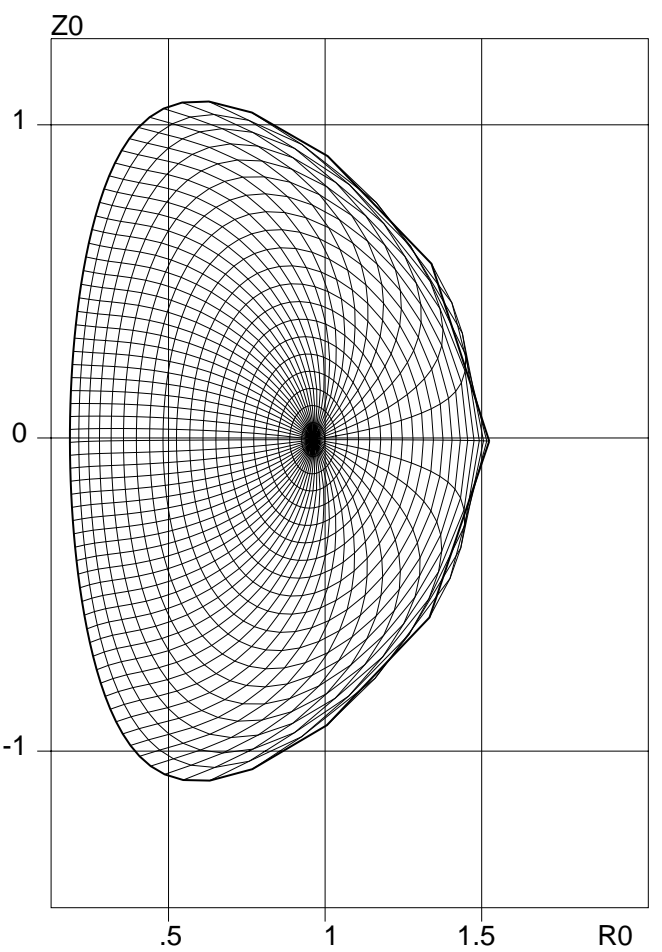

Figure 1: 


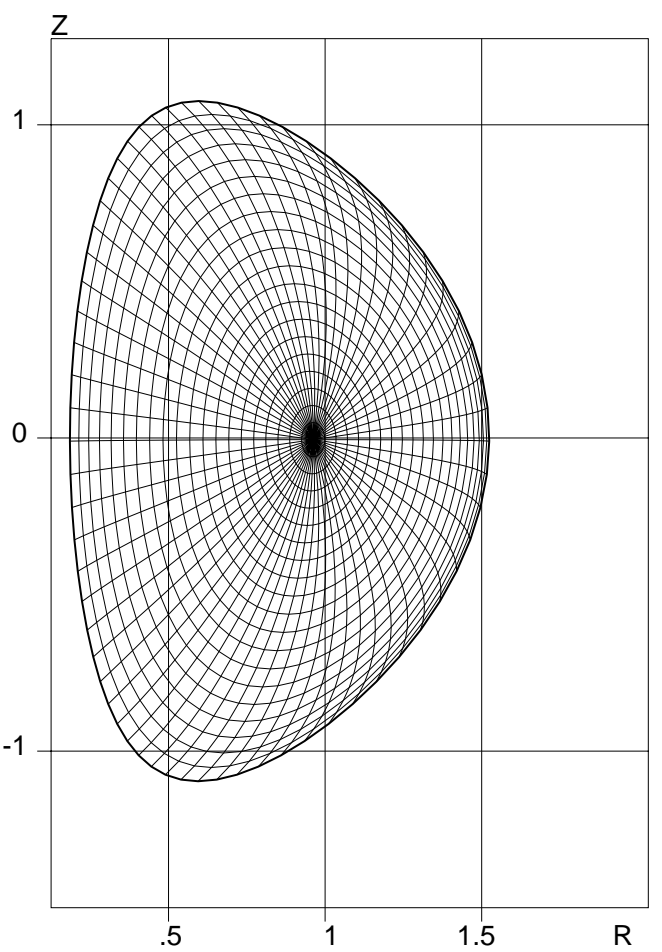

Figure 2: 

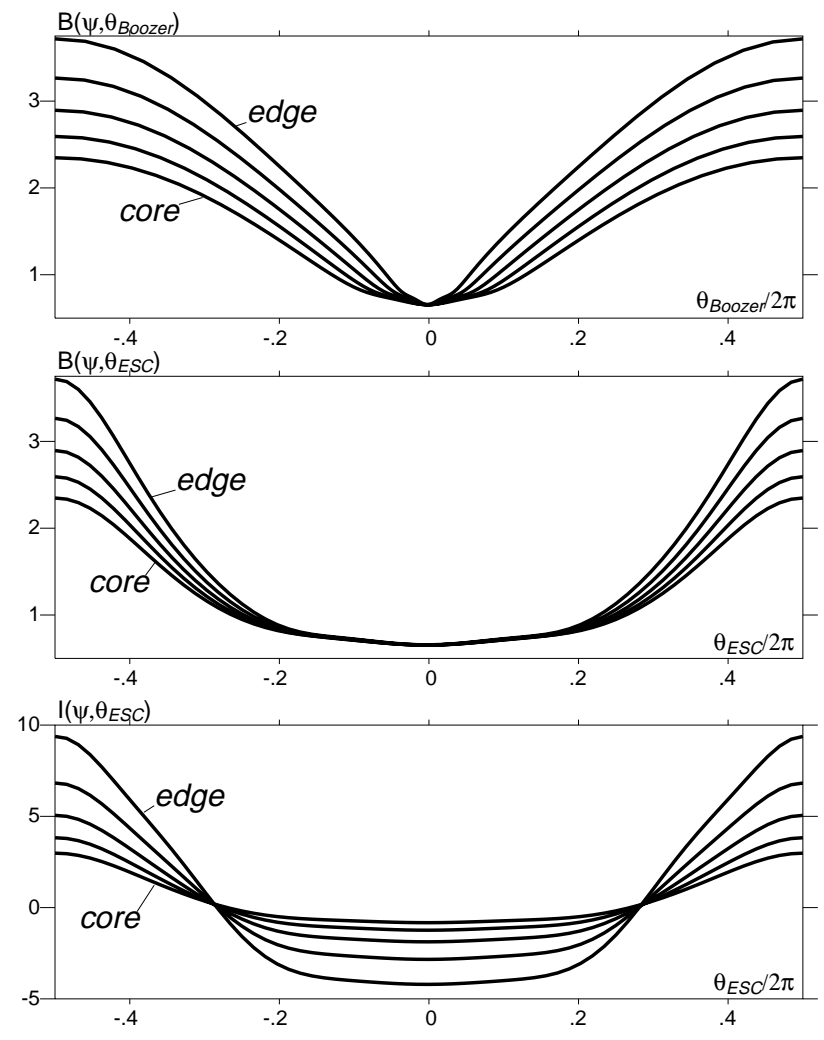

Figure 3: 


\section{External Distribution}

Plasma Research Laboratory, Australian National University, Australia

Professor I.R. J ones, Flinders University, Australia

Professor J oão Canalle, Instituto de Fisica DEQ/IF - UERJ , Brazil

Mr. Gerson O. Ludwig, Instituto Nacional de Pesquisas, Brazil

Dr. P.H. Sakanaka, Instituto Fisica, Brazil

The Librarian, Culham Laboratory, England

Library, R61, Rutherford Appleton Laboratory, England

Mrs. S.A. Hutchinson, JET Library, England

Professor M.N. Bussac, Ecole Polytechnique, France

Librarian, Max-Planck-Institut für Plasmaphysik, Germany

J olan Moldvai, Reports Library, MTA KFKI-ATKI, Hungary

Dr. P. Kaw, Institute for Plasma Research, India

Ms. P.J . Pathak, Librarian, Insitute for Plasma Research, India

Ms. Clelia De Palo, Associazione EURATOM-ENEA, I taly

Dr. G. Grosso, Instituto di Fisica del Plasma, Italy

Librarian, Naka Fusion Research Establishment, J AERI, J apan

Library, Plasma Physics Laboratory, Kyoto University, J apan

Research Information Center, National Institute for Fusion Science, J apan

Dr. O. Mitarai, Kyushu Tokai University, J apan

Library, Academia Sinica, Institute of Plasma Physics, People's Republic of China

Shih-Tung Tsai, Institute of Physics, Chinese Academy of Sciences, People's Republic of China

Dr. S. Mirnov, TRINITI, Troitsk, Russian Federation, Russia

Dr. V.S. Strelkov, Kurchatov Institute, Russian Federation, Russia

Professor Peter Lukac, Katedra Fyziky Plazmy MFF UK, Mlynska dolina F-2, Komenskeho Univerzita, SK-842 15 Bratislava, Slovakia

Dr. G.S. Lee, Korea Basic Science Institute, South Korea

Mr. Dennis Bruggink, Fusion Library, University of Wisconsin, USA

Institute for Plasma Research, University of Maryland, USA

Librarian, Fusion Energy Division, Oak Ridge National Laboratory, USA

Librarian, Institute of Fusion Studies, University of Texas, USA

Librarian, Magnetic Fusion Program, Lawrence Livermore National Laboratory, USA

Library, General Atomics, USA

Plasma Physics Group, Fusion Energy Research Program, University of California at San Diego, USA

Plasma Physics Library, Columbia University, USA

Alkesh Punjabi, Center for Fusion Research and Training, Hampton University, USA

Dr. W.M. Stacey, Fusion Research Center, Georgia Institute of Technology, USA

Dr. J ohn Willis, U.S. Department of Energy, Office of Fusion Energy Sciences, USA

Mr. Paul H. Wright, Indianapolis, Indiana, USA 
The Princeton Plasma Physics Laboratory is operated by Princeton University under contract with the U.S. Department of Energy.

\author{
Information Services \\ Princeton Plasma Physics Laboratory \\ P.O. Box 451 \\ Princeton, NJ 08543
}

Phone: 609-243-2750

Fax: 609-243-2751

e-mail: pppl_info@pppl.gov

Internet Address: http://www.pppl.gov 